\title{
WEBVIRT - FERRAMENTA DE GERENCIAMENTO DE MÁQUINAS VIRTUAIS
}

\author{
WEBVIRT - SOFTWARE TO MANAGE VIRTUAL MACHINES \\ Ronaldo Rathis Sacco', Marco Antônio Sandini Trentin ${ }^{2}$ \\ ${ }^{1}$ Acadêmico do curso de Ciência da Computação e bolsista Pibic/UPF, Universidade de Passo Fundo - UPF, \\ E-mail: contato@ronaldosacco.com.br \\ ${ }^{2}$ Professor do curso de Ciência da Computação, Universidade de Passo Fundo - UPF, E-mail: trentin@ upf.br
}

\begin{abstract}
RESUMO
O conceito de virtualização é bastante conhecido no mundo da TI. Porém, embora o número de empresas que vêm utilizando este conceito esteja em constante crescimento, estima-se ainda que menos da metade das médias e grandes empresas brasileiras realmente se beneficiam desta tecnologia, que tem por principal objetivo possibilitar que um único servidor possa hospedar diversos sistemas operacionais de forma virtualizada. Tendo em vista esse cenário, este trabalho descreve diferentes métodos de virtualização visando facilitar o entendimento e a escolha do método mais indicado para cada caso. Também são apresentadas as vantagens e desvantagens de se utilizar a virtualização nas empresas. Ainda, com o intuito de facilitar o uso da virtualização e ajudar no crescimento de sua utilização, foi desenvolvido um software gratuito e Open Source, chamado de WebVirt. Este software, capaz de gerenciar e monitorar máquinas virtuais, é apresentado detalhadamente neste artigo, assim como seu desenvolvimento e modo de funcionamento. Percebeu-se que com o uso do WebVirt muitas tarefas foram otimizadas, possibilitando a ativação de forma simples e rápida de máquinas virtuais.

Palavras-chave: Virtualização; Hipervisor; WebVirt; Máquina virtual.
\end{abstract}

\begin{abstract}
The concept of virtualization is already well known in the IT world. The number of companies who have been using this concept is steadily growing, but yet it is estimated that less than a half of large and medium size companies really have some benefit from this tecnology, whose main purpose is enable a single server hosts multiple virtualized operating systems. With this information, this work will describe the different virtualizations method for each case. We also report the advantages and disadvantages of using the virtualization in enterprises. Yet in order to facilitate the use of virtualization and help grow their use, has created a free and open source software called WebVirt. This software can manage and monitor virtual machines and that WebVirt will be presented in detail in this paper, as well as its development and operation. It was felt that with the use WebVirt, several tasks have been optimized, allowing the simple and fast activation of a virtual machine.
\end{abstract}

Keywords: Virtualization; Hypervisor; Webvirt; virtual machines.

\section{INTRODUÇÃO}

Há poucos anos, era comum em empresas de médio e grande porte a existência de Centros de Processamentos de Dados, também conhecidos como CPDs, onde imperavam os grandes mainframes. Estes, dotados de diversas CPUs e vários MegaBytes de memória, eram responsáveis por um consumo considerável de energia elétrica e de espaço físico.

Hoje ainda é comum encontrar um servidor ativo para cada tipo de serviço. É muito comum ainda nas empresas existir um servidor físico ligado para seu sistema interno e outro, para servidor web. Esses são ligados a um terceiro servidor, que faz a conexão com a internet, atuando como um 
firewall. Como se pode perceber, o que ocorre normalmente é um desperdício significativo de hardware.

Segundo Márquez (2010), gerente da Enterprise Storage, estatísticas mostram que são aproveitados cerca de $10 \%$ a $30 \%$ do poder de processamento dos servidores, ou seja, "sobram $70 \%$ de recursos ou talvez até mais, nos servidores, em alguns casos".

Virtualizar plataformas em um único servidor pode ser benéfico, e são vários os benefícios de reduzir a quantidade de servidores virtualizando-os. Uma quantidade menor de servidores reduz, além do espaço físico, o consumo de energia, custos com resfriamento e gerenciamento. Menos hardware também significa maior confiabilidade. Em suma, a virtualização traz vantagens técnicas como também vantagens em termos financeiros, enquadrando-se nos conceitos de Green IT, também conhecido por "tecnologia verde".

O Green IT visa criar formas ideais para trabalhar com o avanço tecnológico juntamente com o meio ambiente. "[...] a TI verde, além de indicar um posicionamento politicamente correto, significa economizar recursos, cortar custos, o que em tempos de crise é questão de sobrevivência". (BARROS, 2009).

Hoje, com os avanços da tecnologia, os processadores estão cada vez mais rápidos. Se comparados com o passado, consegue-se aumentar significativamente o poder computacional dos processadores por realizarem muito mais cálculos, utilizarem menos energia elétrica e, o melhor, com tamanho cada vez menor. Isso levou a que grandes empresas, como a Citrix, VMware e Microsoft, investissem fortemente em pesquisas sobre virtualização.

De uma perspectiva empresarial,

\begin{abstract}
pode-se alcançar uma redução dos custos totais de propriedade (TCO - Total Cost of Ownership), pois o hardware é melhor aproveitado quando múltiplos sistemas operacionais coexistem em uma mesma máquina física. Imagine executar apenas duas máquinas virtuais em cada servidor que uma empresa possui. Isso poderia representar que apenas $50 \%$ do hardware seria necessário para uma infraestrutura de computação equivalente. (MATHEWS et al., 2009, p.5)
\end{abstract}

Segundo Morimoto (2009), o uso de virtualização permite agrupar diversos servidores de baixa demanda em uma única máquina. Caso se opte por manter o servidor DNS ou o servidor de email em uma máquina separada por questões de segurança, pode-se muito bem utilizar uma máquina virtual em vez de utilizar um segundo servidor dedicado.

Existem diversos tipos e modos de virtualização. Alguns deles possuem alterações diretamente no kernel, visando a um melhor desempenho; outros buscam a facilidade de virtualizar, podendo ser instalados e executados com apenas alguns passos, como é o caso do VirtualBox da empresa Oracle.

Uma das camadas mais importantes da virtualização é a camada do hypervisor, como pode ser visualizado na Figura 1. Esta camada é responsável por fornecer plataformas de hardware virtual isoladas para execução, que, por sua vez, dão uma ilusão de acesso total à máquina subjacente a uma camada intermediária entre o software (Sistema Operacional - S.O.) e o hardware. Assim, os hypervisors são responsáveis por alocar recursos do hardware real para cada hardware virtual. 


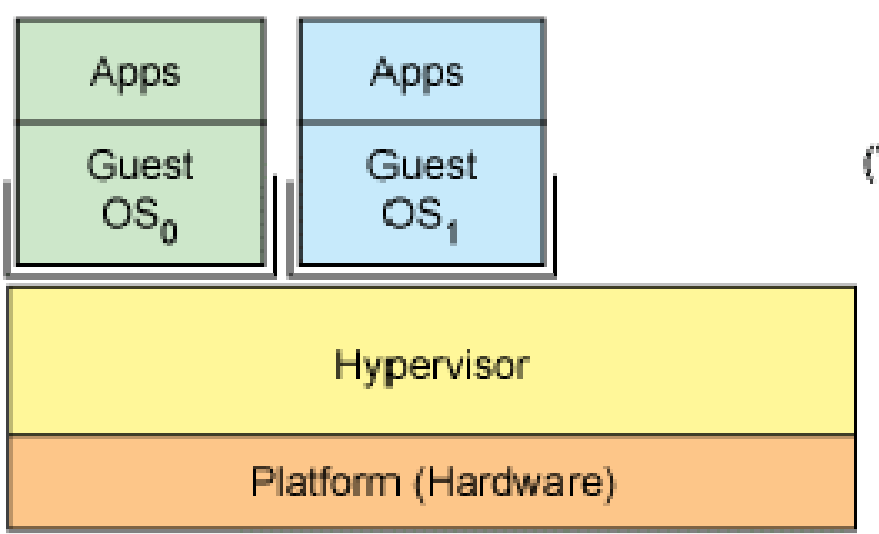

\section{Virtual machines \\ (Virtual Hardware users)}

\section{Vitual hardware}

\section{Physical hardware}

Figura 1 - Arquitetura simples em camada

Fonte: www.vmware.com

O hypervisor fica, então, responsável por gerenciar toda a comunicação entre as máquinas virtuais (virtual machines, ou simplesmente VMs) e o hardware físico, incluindo IRQs, endereços de memória, processamento, IO, etc., de forma que cada S.O. virtualizado "imagine" estar rodando diretamente sobre um hardware físico.

Como se pode perceber, o hypervisor lida com questões de extrema importância para o S.O., sendo o grande responsável pelo desempenho das VMs. Segundo Jones (2009), uma das mais importantes inovações do Linux é a sua transformação em um hypervisor (ou seja, um S.O. para outros S.O.). Várias soluções de hypervisor lançadas fazem uso do Linux como núcleo.

O objetivo deste artigo é apresentar o estudo realizado sobre VMs e o desenvolvimento de um sistema para gerenciar VMs, chamado WebVirt. A criação deste sistema possibilita que empresas de qualquer porte possam se beneficiar com o uso do WebVirt, buscando facilidade de criação, gerenciamento e monitoramento de VMs.

\section{WEBVIRT}

O WebVirt é um projeto OpenSource, criado com o intuito principal de facilitar o gerenciamento de máquinas virtuais. Para isso algumas questões, tais como usabilidade, agilidade e autonomia, foram cuidadosamente implementadas neste software.

O projeto apresentado será dedicado ao "WebVirt for Vmware Server", desenvolvido para funcionar em paralelo com o Vmware Server. O WebVirt permite a configuração para outras formas de virtualização, tais como o Xen ("WebVirt for Xen").

Além do objetivo principal de facilitar o gerenciamento de VMs, o WebVirt garante a correta configuração de cada máquina virtual, não exigindo dos usuários conhecimentos aprofundados em Linux, bem como em VMware, pois para realizar a ativação de uma máquina virtual o VMware é totalmente transparente para o usuário, não sendo necessário escrever qualquer linha de comando após a instalação do WebVirt ter sido concluída.

O sistema é composto por seis módulos:

1) Sistema Operacional;

2) Sistema Hospedeiro;

3) Interface $W e b$;

4) Configuração de templates;

5) Sistema gerador das máquinas virtuais;

6) Serviço de monitoramento das máquinas virtuais. 
O WebVirt permite a utilização por diversos usuários, mas apenas um tem poder de administrador. $\mathrm{O}$ usuário com permissões de administrador pode:

- monitorar todas as máquinas virtuais criado pelos demais usuários;

- criar novos usuários;

- definir configurações essenciais para o correto funcionamento do sistema;

- adicionar templates para as novas máquinas virtuais.

Os usuários clientes limitam-se a:

- criar novas máquinas virtuais;

- definir espaço em disco, IP, memória e S.O. para cada VM;

- reiniciar uma VM;

- realizar backups (também chamado de snapshot);

- restaurar o snapshot.

O usuário administrador, ao contrário dos usuários clientes, não tem permissão para criar máquinas virtuais, pois a ideia é que apenas os usuários clientes criem VMs e o usuário com poderes administrativos possa controlar apenas questões de configurações.

A principal razão da possibilidade de criação de vários usuários no WebVirt é permitir que organizações com vários funcionários não necessitem compartilhar o mesmo usuário e senha entre todos. Dessa forma, é possível que cada usuário controle a sua VM e o administrador do WebVirt tenha controle sobre todas as VMs.

$\mathrm{O}$ WebVirt trabalha com templates, ou seja, para inicializar uma nova VM um template deve ter sido pré-configurado. Um template nada mais é que uma VM instalada, configurada, compactada e agrupada por "tar.gz" e pronta para ser utilizada. Este template servirá como base para a criação das demais VMs. Por exemplo, pode-se criar um template com sistema operacional Debian e, a partir deste, gerar várias VMs iguais a este template.

O WebVirt foi projetado para suportar vários templates, limitado apenas pela capacidade do hardware. Podem ser criados, por exemplo, templates diferentes: um template Windows, um Ubuntu e um CentOS; ou criar vários templates do mesmo S.O., diferenciando-os apenas pela versão do mesmo e/ou diferenciando os programas pré-instalados em cada um deles.

A Figura 2 ilustra o gerenciamento das VMs utilizando o WebVirt. 


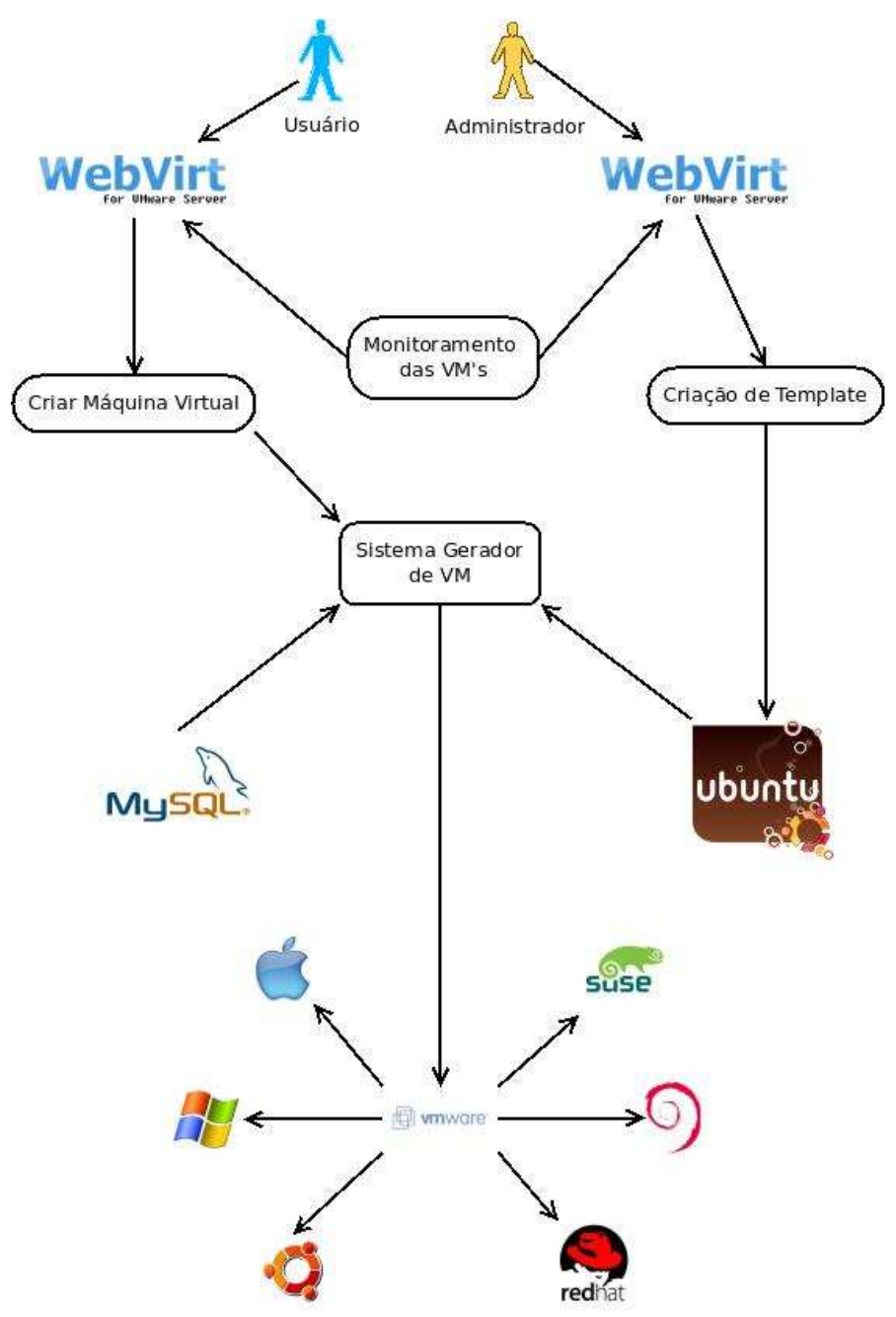

Figura 2 - Descrição do WebVirt

\subsection{Requisitos Necessários e Instalação}

O WebVirt necessita para seu correto funcionamento ser executado em um S.O. Linux com um serviço Web instalado. Recomenda-se a utilização do S.O. Debian, além do Apache, PHP5 e MySQL. Os requisitos mínimos de hardware desse computador são 1GB de memória RAM, processador de 2.0 $\mathrm{GHz}$ dual-core e placa de rede Fast Ethernet.

Os resultados da instalação e avaliações do WebVirt apresentados neste artigo foram obtidos a partir de um servidor HP ProLiant ML110, utilizando Debian como S.O., com processador Intel Pentium 4 Processor 630, 3.0GHz e 4GB de memória RAM.

A instalação do WebVirt ${ }^{1}$ é bastante fácil até mesmo para usuários leigos. A Figura 3 apresenta um printscreen de todo o processo de instalação:

\footnotetext{
${ }^{1} \mathrm{O}$ download do WebVirt, bem como seu código fonte, pode ser encontrado em http://webvirt.ronaldosacco.com.br.
} 


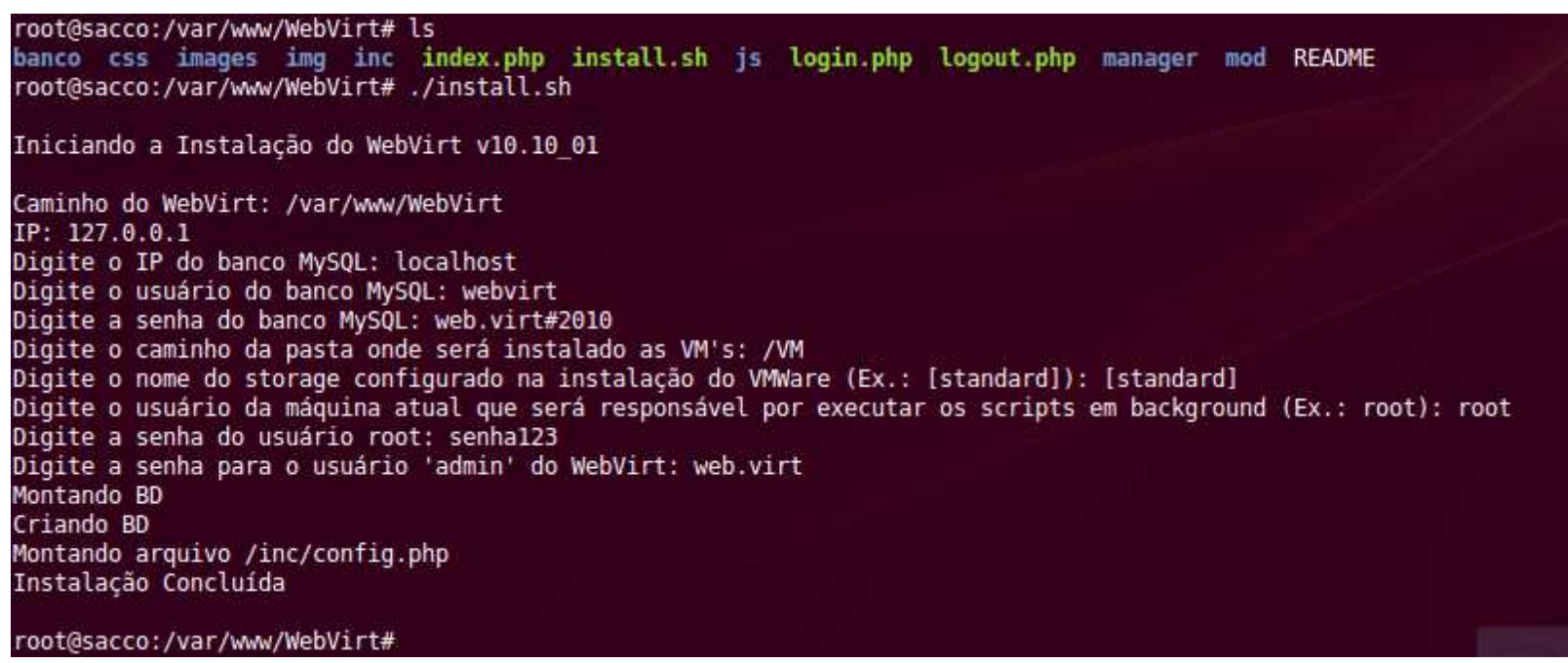

Figura 3 - Demonstração da instalação do WebVirt

Após ter sido concluída com sucesso a instalação conforme a figura acima, basta acessar pelo navegador o site do WebVirt localizado na máquina de instalação. O usuário e senha solicitados são os do administrador, o mesmo configurado na instalação.

\section{WEBVIRT PARA USUÁRIOS CLIENTES}

Neste capítulo é apresentado o sistema por meio do qual é possível criar e gerenciar VMs. Por padrão, nenhuma conta de usuário cliente é definida na instalação. Para isso é necessário ser criado um novo usuário cliente na área de administração do WebVirt. A Figura 4 mostra a página principal onde é possível realizar a criação de VMs pelo WebVirt.

Nesta tela o usuário deve cadastrar algumas informações a respeito da VM que deseja ser criada. Começando pelo "Nome VM", é o campo onde é definido o nome para a VM. Este nome serve apenas para identificar a VM para o próprio usuário. O nome escolhido como exemplo foi "Máquina de Testes".

Os quatro campos seguintes são referentes à configuração da VM, como S.O., IP, memória e espaço em que deverá ser alocado para esta VM. No exemplo da Figura 4 está selecionado no campo Sistema Operacional "Debian Etch 4.0 (deb.pwd2010)". Este campo possui duas informações: a primeira é o S.O. que será virtualizado; a segunda, entre parênteses, informa a senha deste S.O.. É com esta senha que o cliente do WebVirt acessará a máquina via SSH depois que estiver criada.

O segundo campo mostra o IP que será criado para esta VM. Por padrão, as VMs estão configuradas sem nenhum IP, apenas com o DHCP ativado, ou seja, a VM, quando ativada, irá solicitar um IP para ela na rede.

O WebVirt possui um script funcionando em background que controla o serviço de DHCP. Portanto, quando a VM inicializar, o WebVirt estará preparado para entregar um IP para a VM utilizar. Este IP é definido automaticamente de acordo com o MAC da placa de rede da VM. Esse processo é transparente para o usuário final, porém é importante saber que tais IP's mostrados neste campo são definidos pelo administrador do WebVirt. Portanto, a alteração do IP da VM pode fazer com que ela pare de funcionar, assim como a alteração do MAC da placa de rede. 


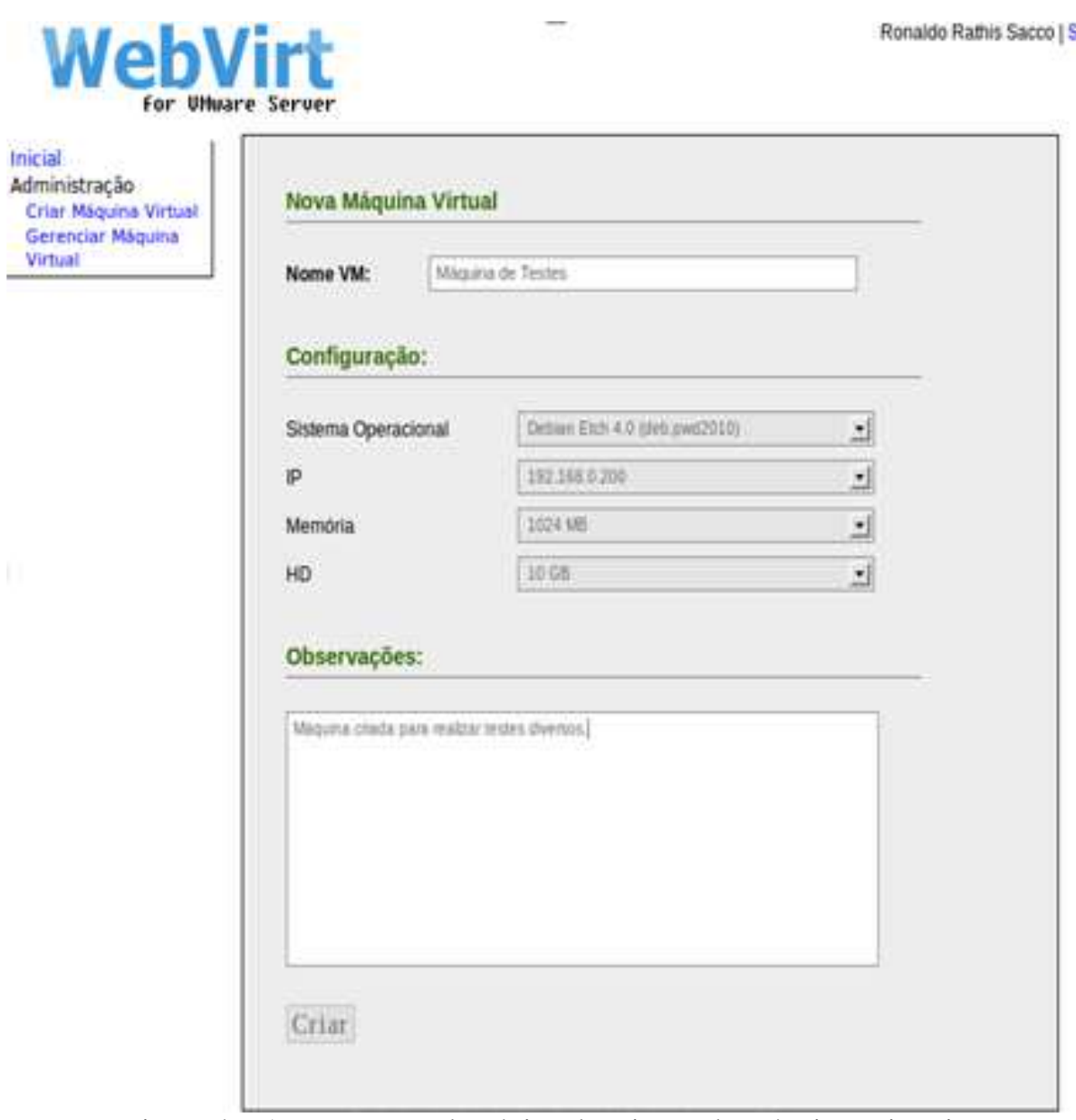

Figura 4 - Apresentação da página de criação de máquinas virtuais

Após preenchidas as informações necessárias para a criação de uma VM, basta clicar em "Criar". A VM será automaticamente criada e disponibilizada para acesso via SSH (Linux), ou pela Área de trabalho remota (Windows). Esse processo leva, em média, 2 minutos para ser concluído, dependendo muito do processador do hardware. Nos testes realizados a VM levou menos de 1 minuto para ser ativada, ficando pronta para uso.

A administração das VMs pode ser encontrada em "Gerenciar Máquina Virtual”, conforme mostra a figura acima.

Na Figura 4 pode-se verificar a existência de duas VMs ativas: "Canall Asterisk" e "Ronaldo Sacco"; ambas possuem como S.O. o Debian Etch 4.0. Outras informações referentes a essas VMs também são descritas nessa figura, como IP utilizado, quantidade de memória RAM, capacidade do disco rígido e percentual utilizado. Por fim, mostra-se quando foi realizado o último backup, também conhecido por snapshot da VM.

Um snapshot é uma cópia total da VM no estado em que ela parou. É realizado um backup de disco, memória, BIOS e dados em geral. Este backup pode ser restaurado a qualquer momento, voltando a VM ao estado original do último snapshot. Esse procedimento é bastante utilizado em caso de testes de novos programas ou sistemas em que ocorre um grande risco de travamento do sistema ou perda de dados importantes. Dessa forma, fica fácil voltar ao estado original da máquina virtual em poucos minutos.

A realização de snapshot é uma das grandes vantagens que a virtualização de S.O. proporciona para o usuário, pois a mesma tarefa, caso fosse necessário executar em uma máquina física, levaria tempo e trabalho muito maior, em comparação com a mesma tarefa realizada em uma máquina virtualizada. 


\section{WebVirt}
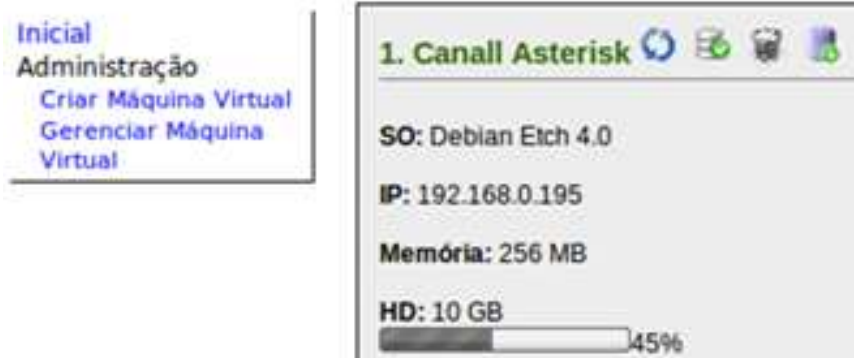

Figura 5 - Apresentação da página de Gerenciamento de VMs

A criação de snapshot é realizada pelo usuário. O usuário cliente pode gerar apenas um snapshot da sua VM a qualquer momento. O WebVirt propicia esta funcionalidade de forma automatizada na tela de gerenciamento das VMs. Nesta tela é apresentada a data e hora de apenas o último snapshot gerado para aquela VM, pois os snapshot's não são cumulativos, ou seja, não é salvo um histórico de backups na máquina virtualizadora.

O primeiro botão apresentado na Figura 4 serve para reiniciar a VM. A reinicialização da VM será forçada, ou seja, é diferente de enviar um comando para o S.O. "avisando" para que seja desligado, pois neste caso a VM terá seu processamento interrompido. É como se fosse, comparado com uma máquina física, pressionado o botão de reiniciar.

$\mathrm{O}$ recurso de reiniciar a VM é bastante utilizado em dois casos: no primeiro, quando a VM entra em loop, ou algum processo trava por algum motivo; no segundo, quando a VM estiver desligada. Por exemplo, caso uma VM Linux esteja sendo acessada e o comando halt, que serve para desligar a VM, for executado, a máquina estará virtualmente desligada. Esta máquina virtual deixará de consumir recursos da máquina hospedeira, como processamento e memória, porém ficará pronta para voltar ao funcionamento em qualquer momento. Basta clicar no botão de "reiniciar" que a máquina será novamente ativa.

O segundo botão apresentado na Figura 4 realiza um snapshot da VM. A criação do snapshot não interrompe o funcionamento da VM.

O terceiro botão realiza a exclusão da VM. Esta exclusão retira esta VM da listagem e exclui todos os arquivos e informações referentes a ela. Esse processo demora cerca de 1 minuto para ser executado e não existe atualmente forma para recuperar em caso de acidente. 
Quando uma VM é excluída, o IP que utilizava volta para a "lista" de IPs disponíveis, podendo uma nova VM ser criada com o mesmo IP da antiga VM.

O quarto e último botão ativa a restauração da VM a partir de seu snapshot. Para que esse procedimento funcione é necessário que exista algum snapshot criado a fim de que seja possível voltar a VM a um estado anterior.

$\mathrm{O}$ processo de restauração do backup, ou snapshot, interrompe por alguns minutos o funcionamento da VM, pois exige a parada temporária da mesma, seguido da exclusão de todos os arquivos, descompactação, restauração do backup anterior para, então, ativar a mesma.

De acordo com os testes realizados, o procedimento de restauração de bakcups demorou cerca de 3 minutos para ser completamente executado.

\section{WEBVIRT PARA USUÁRIOS ADMINISTRADORES}

Para o usuário administrador do sistema, o WebVirt possui quatro telas de configuração. São elas: Hosts, IPs Liberados, Usuários e VMs. A configuração do host da máquina hospedeira é realizado pelo menu Hosts, conforme apresentado na Figura 6.

\section{WebVirt \\ Administrador / Sair}

\begin{tabular}{l} 
Inicial \\
Administração \\
Hosts \\
IPs Liberados \\
Usuários \\
VMs \\
\hline
\end{tabular}

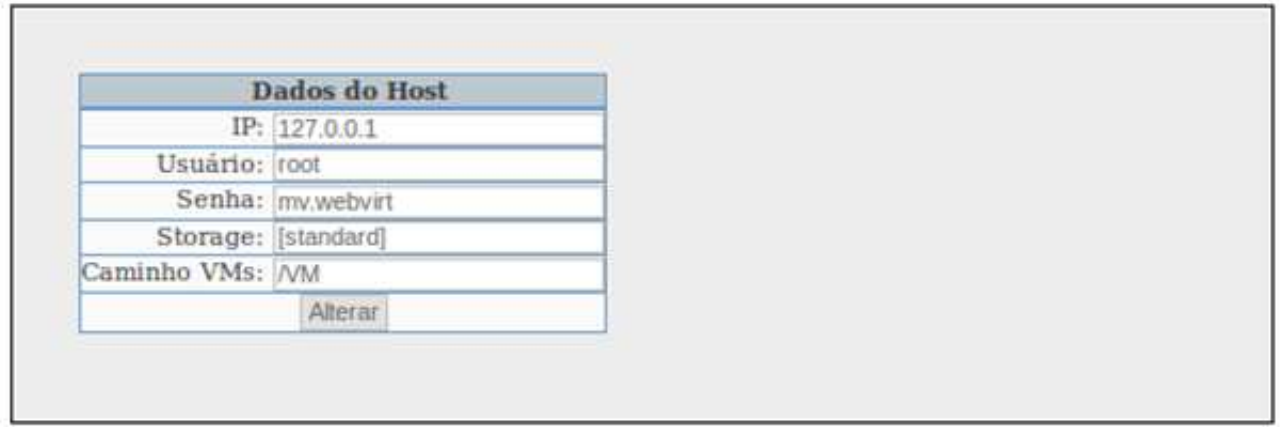

Figura 6 - Apresentação do menu hosts

Este menu, embora seja chamado de "Hosts", configura apenas um host: a máquina virtualizadora. Os fontes do WebVirt foram criados para suportar, de maneira que não seja necessária a adaptação de grande parte do código fonte, um módulo de Inteligência Artificial - IA capaz de trabalhar com mais de uma máquina virtualizadora. Esse procedimento é totalmente transparente para o usuário cliente. A ideia, ainda em projeto, é que, quando um usuário cliente escolher a criação de uma VM, este sistema inteligente seja capaz de identificar qual máquina virtualizadora é a mais adequada para rodar o S.O. com os requisitos especificados.

Como o WebVirt é OpenSource, espera-se que algum colaborador possa vir a desenvolver esta facilidade (desenvolvimento de um módulo de I.A) e adapte-a ao WebVirt, para que esta nova funcionalidade possa ser utilizada em um futuro próximo.

Em "IPs Liberados" encontram as configurações de IPs que serão liberados para as VMs, conforme mostra a Figura 7. As duas colunas que aparecem nessa figura representam os IPs disponíveis e IPs em uso. A primeira coluna, ao contrário da segunda, é editável, ou seja, cabe ao usuário administrador definir quais IPs ficarão disponíveis ao usuário cliente. A segunda coluna é apenas um campo readonly, no qual ficam os IPs que estão em utilização por alguma VM. Assim, esses IPs não poderão ser alterados nem pelo administrador do WebVirt até que a VM do respectivo IP seja excluída ou tenha seu IP alterado. 


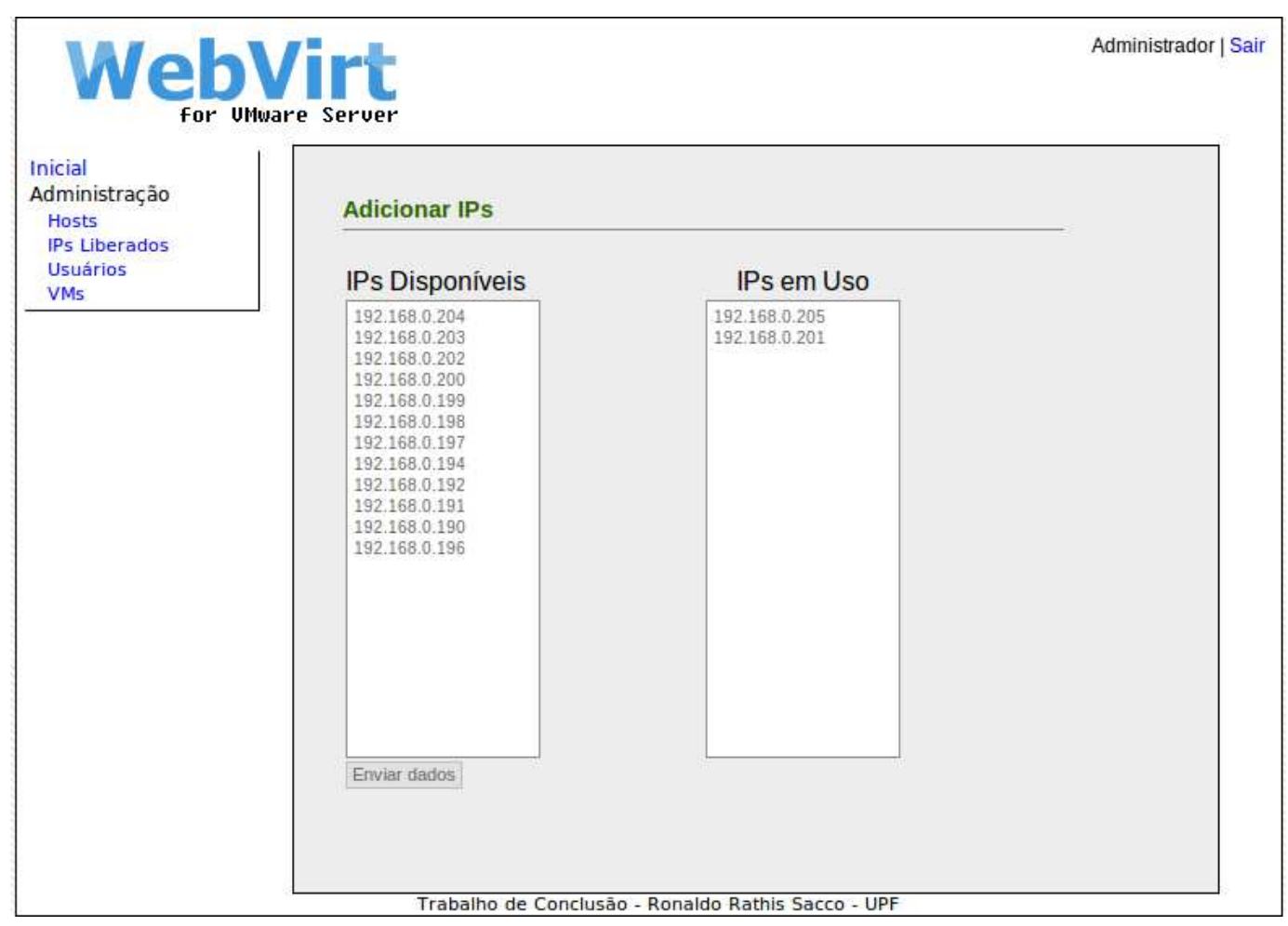

Figura 7 - Apresentação do menu IPs Liberados

No menu "Usuários" podem ser criados novos usuários clientes. O menu "VMs" contém informações dos templates criados e configurados para serem exibidos na área do usuário cliente. Depois de configurado, todos os usuários clientes cadastrados no sistema poderão visualizar o template criado. A Figura 8 mostra uma captura de tela retirada da janela de configuração dos templates.

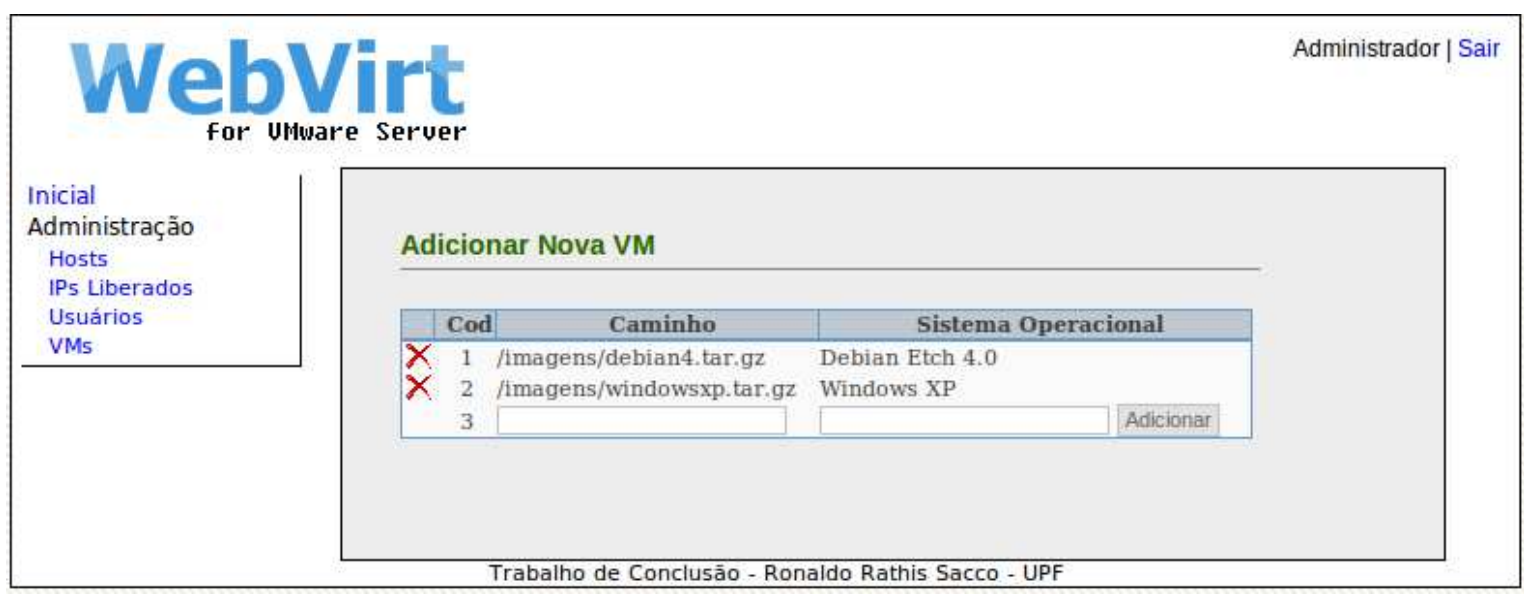

Figura 8 - Apresentação do menu VMS

\section{CONCLUSÃO}

Atualmente, com o alto poder computacional, se comparado com computadores de poucos anos atrás, pode-se facilmente se beneficiar da virtualização. Hoje é possível encontrar diversos 
softwares de virtualização que permitem que profissionais da área de informática pensem em formas inovadoras de projetar uma arquitetura de servidores, tendo como foco buscar o melhor aproveitamento do uso do hardware e diminuição de custos sem perder a qualidade do serviço e, inclusive, podendo oferecer um serviço de melhor qualidade.

O WebVirt foi criado com código OpenSource e ficará disponível para download, junto com seu código fonte, para quem desejar fazer uso e/ou efetuar melhorias em seu código. Tal sistema mostrou-se compatível e estável quando utilizado em conjunto com o Vmware Server. Todo o código do funcionamento do WebVirt está pronto, e a adaptação para suportar novos softwares de virtualização pode ser facilmente realizada com poucas alterações em seu código fonte.

Esta ferramenta foi testada em uma empresa de porte médio e atendeu satisfatoriamente à necessidade desse empreendimento, no que diz respeito à criação e ao gerenciamento de diferentes VMs por diferentes funcionários. Sua facilidade de uso e rapidez é notória, afirmação essa baseada na aprovação relatada pelos usuários.

Com a criação desse sistema pode-se concluir que, embora ainda seja precária a documentação existente para criar a comunicação entre um software e um sistema de gerenciamento de VMs, é possível, de maneira fácil e rápida, criar aplicativos capazes de se comunicar com as VMs e, então, realizar seu gerenciamento, monitoramento, ações e/ou tomadas de decisões de acordo com a necessidade de cada administrador. Como trabalhos futuros espera-se adaptar o WebVirt para que funcione não somente com o Vmware, mas também com o Xen. Uma vez que o código-fonte do WebVirt encontra-se disponível e acessível, espera-se que este trabalho seja continuado e adaptado para que possa ser instalado por um simples apt-get install.

\section{REFERÊNCIAS BIBLIOGRÁFICAS}

A PERFORMANCE comparison of Hypervisors. Disponível em: 〈http://www.vmware.com/pdf/hypervisor_performance.pdf>. Acesso em: 25 jul. 2010.

BARROS, A. Virtualização: TI verde e economia além do discurso. 2009. Disponível em: $<$ http://olhardigital.uol.com.br/produtos/mobilidade/celulares-verdes-sao-uma-alternativa-paradiminuicao-do-lixo-eletronico/8728>. Acesso em: 15 jul. 2010.

BUENO, H. Virtualização - Um pouco de história. 2009. Disponível em: <http://hbueno.wordpress.com/2009/04/29/virtualizacao-um-pouco-de-historia/>. Acesso em: 20 jul. 2010 .

COELHO, F. Virtualização VMWare e Xen. 2008. Disponível sem: <http://www.gta.ufrj.br/grad/09_1/versao-final/virtualizacao>. Acesso em: 20 ago. 2010.

COMPARAÇÃO entre benchmarks da XenSource e Vmware. Disponível em: <http://wiki.xenbr.org/index.php?title=Compara\%C3\%A7\%C3\%A3o_entre_benchmark_Xensource_e_Vmware>. Acesso em: 2 out. 2010.

GREEN Calculator. Disponível em: 〈http://www.VMware.com/solutions/green/calculator.html>. Acesso em: 17 out. 2010.

HYPERVISOR Performance Comparison. Disponível em: <http://blogs.xensource.com/simon/wpcontent/uploads/2007/03/hypervisor_performance_comparison_1_0_3_no_esx-data.pdf >. Acessado em: 10 set. 2010 . 
JONES, T. Anatomia de um Hypervisor Linux. 2009. Disponível em:

<http://www.ibm.com/developerworks/br/library/l-hypervisor/>. Acesso em: 25 set. 2010.

LEONEL, T. Ubuntu 10.04 com VMware Server 2.0.x. 2010. Disponível em:

<http://www.vivaolinux.com.br/dica/Ubuntu-10.04-com-VMware-Server-2.0.x >. Acesso em: 12 jul. 2010 .

MARQUEZ, O. Por que virtualizar?, Disponível em: <http://h20341.www2.hp.com/ eNewsletter/downloads/Revista_Adaptive_VIII_port (2).pdf>. Acesso em: 5 dez. 2010.

MATHEWS, J. et al. Executando o Xen. Rio de Janeiro: Alta Books, 2009.

MORIMOTO C.E. Servidores Linux, guia prático. Porto Alegre: Sul Editores, 2008.

TANENBAUM, A.S. Redes de computadores. Rio de Janeiro: Campus, 1997.

VMWARE SERVER MASTER END USER LICENSE AGREEMENT. Disponível em: <http://register.VMware.com/content/eula.html>. Acesso em: 2 nov. 2010.

XEN Wiki. Disponível em: <http://wiki.xensource.com/xenwiki/>. Acesso em: 7 jun. 2010. 